\title{
Convergence of a Collocation Scheme for a Retarded Potential Integral Equation
}

\author{
Dugald B Duncan ${ }^{1}$ and Penny J Davies ${ }^{2}$ \\ 1 Department of Mathematics, Heriot-Watt University, Riccarton, Edinburgh, \\ EH14 4AS, UK (D.B.Duncan@ma.hw.ac.uk) \\ 2 Department of Mathematics, University of Strathclyde, 26 Richmond St, Glasgow, \\ G1 1XH, UK
}

\begin{abstract}
Time domain boundary integral formulations of transient scattering problems involve retarded potential integral equations (RPIEs). We outline how Fourier and Laplace transforms can be used to obtain an $O\left(h^{2}\right)$ convergence result for a "polar" piecewise-linear collocation approximation of a scalar RPIE on an infinite flat plate.
\end{abstract}

\section{Introduction}

We consider the approximate solution of the scalar RPIE

$$
\int_{\Gamma} \frac{u\left(\boldsymbol{x}^{\prime}, t-\left|\boldsymbol{x}-\boldsymbol{x}^{\prime}\right|\right)}{\left|\boldsymbol{x}-\boldsymbol{x}^{\prime}\right|} d \boldsymbol{x}^{\prime}=a(\boldsymbol{x}, t) \quad \text { for } \boldsymbol{x} \in \Gamma, \quad t \in(0, T)
$$

for $u$ when $a$ is given on $\Gamma \times(0, T)$ for fixed $T>0$, and

$$
u \equiv 0, \quad a \equiv 0 \quad \text { for all } t \leq 0 .
$$

This is the single layer potential equation for acoustic scattering from the surface $\Gamma$ [8, Sect. 2.3]. It is an important problem in its own right, and also appears as a part of more complicated electromagnetic and elastodynamics problems.

Various numerical methods for approximating RPIEs have been reported in the literature. For example a full Galerkin approximation in time and space is described and analysed in [1,7,9], and collocation schemes for the problem have been described by many authors including $[10,11]$. Collocation schemes are generally easier to implement and harder to analyse than variational methods.

Here we examine the collocation scheme described in [5], which uses a local change of variable to polar coordinates. Stability was demonstrated, and a convergence estimate at the (slow!) rate $O(1 / \ln h)$ as mesh size $h \rightarrow 0$ was obtained in [5]. We describe this scheme in $\S 2$ and outline how to obtain the much improved convergence estimate $O\left(h^{2}\right)$ in $\S 3$.

\section{Polar collocation scheme}

We approximate the RPIE (1) on an infinite flat surface in order to make Fourier convergence analysis possible. We discretise it uniformly in space into squares 
of side $h$, with uniform time-step $\Delta t$. The space nodes $\boldsymbol{x}_{\beta}$ are at the corners of the square space mesh and the time nodes are at $t^{n}=n \Delta t$ for integer $n$. The approximation

$$
u(\boldsymbol{x}, t) \approx U(\boldsymbol{x}, t)=\sum_{\beta, s} U_{\beta}^{s} \varphi_{\beta}(\boldsymbol{x}) \psi_{s}(t) .
$$

is piecewise-linear in both space and time.

We substitute $U$ for $u$ in (1) and collocate at all the space nodes $\boldsymbol{x}_{\alpha}$ and time nodes $t^{n}(n=1,2, \ldots)$ to obtain the approximation

$$
\sum_{\beta, s} U_{\beta}^{s} \int_{\Gamma} \frac{\varphi_{\beta}\left(\boldsymbol{x}^{\prime}\right) \psi_{s}\left(t^{n}-\left|\boldsymbol{x}_{\alpha}-\boldsymbol{x}^{\prime}\right|\right)}{\left|\boldsymbol{x}_{\alpha}-\boldsymbol{x}^{\prime}\right|} d \boldsymbol{x}^{\prime}=a\left(\boldsymbol{x}_{\alpha}, t^{n}\right) .
$$

The integrals above involve quite complicated domains which are the intersections of the support of the spatial basis functions $\varphi_{\beta}\left(\boldsymbol{x}^{\prime}\right)$ with the temporal basis functions evaluated at retarded times $\psi_{s}\left(t^{n}-\left|\boldsymbol{x}_{\alpha}-\boldsymbol{x}^{\prime}\right|\right)$. This is quite different from standard finite element problems. The integrals can be computed exactly with some effort (for example using Stokes' theorem) and then appear to produce a stable scheme over a range of mesh ratios, while more straightforward, standard quadrature methods do not $[5,6]$. We use the word "appear" here since the Fourier stability analysis involves a step that must be verified numerically.

An alternative to standard quadrature and exact evaluation is the "polar coordinate" scheme described in [5]. For each spatial collocation point $\boldsymbol{x}_{\alpha}$ in turn, we change variables in the integrals in (4) to polar coordinates $(R, \theta)$ where $R=\left|\boldsymbol{x}_{\alpha}-\boldsymbol{x}^{\prime}\right|$. Keeping the $\theta$ integrals exact, we approximate the $R$ integrals using the trapezoidal rule to get

$$
\Delta t \sum_{s=0}^{n} W_{s}^{n} \sum_{\beta} U_{\beta}^{n-s} \int_{0}^{2 \pi} \varphi_{\beta}\left(\boldsymbol{x}_{\alpha}+R \boldsymbol{e}_{\theta}\right) d \theta=a\left(\boldsymbol{x}_{\alpha}, t^{n}\right) .
$$

where $\boldsymbol{e}_{\theta}=(\cos \theta, \sin \theta)$, and the trapezoidal weights $W_{0}^{n}=1 / 2, W_{n}^{n}=1 / 2$ and $W_{s}^{n}=1$ otherwise. Implementation for finite surfaces is examined in [3] and stability is investigated in [5] using Fourier methods (see also [4]). The polar scheme appears to be stable for all values of the mesh ratio $r$.

\section{Convergence}

We make the following assumptions to carry out our convergence analysis.

Hypotheses. Suppose

(H1) that $a \in H_{*}^{m+2}\left(0, T ; H^{s+2}\left(\mathbb{R}^{2}\right)\right)$ for $s \geq 4$ and (integer) $m \geq 4$ and hence that exact solution $u \in H_{*}^{m}\left(0, T ; H^{s}\left(\mathbb{R}^{2}\right)\right.$ ) (see [6]);

(H2) that the polar collocation scheme is stable at mesh ratio $r=\Delta t / h \in(0, \infty)$.

We need to make (H2) explicitly because stability has only been verified numerically and not proved rigorously in [5]. 
On the infinite flat plate with uniform space and time grids, the approximation (5) can be written as

$$
\sum_{m=0}^{n-1} \mathbb{Q}^{m} U_{j, k}^{n-m}=a_{j, k}^{n},
$$

where $U_{j, k} \approx u(j h, k h)$ and the $\mathbb{Q}^{m}$ are made up of spatial shift operators. They are translation invariant on the space mesh because it is uniform and the surface $\Gamma$ is flat and unbounded. Taking the discrete Fourier transform (DFT) of (6) at frequency $\boldsymbol{\omega}$ yields

$$
\sum_{m=0}^{n-1} q_{m}(h \boldsymbol{\omega}) \tilde{U}^{n-m}(\boldsymbol{\omega})=\tilde{a}^{n}(\boldsymbol{\omega}),
$$

where for any mesh function $\underline{v}=\left(v_{j, k}\right)$ the DFT is defined by

$$
\tilde{v}(\boldsymbol{\omega})=h^{2} \sum_{j, k} v_{j, k} e^{-i\left(x_{j}, y_{k}\right) \cdot \boldsymbol{\omega}} \quad \text { for } \boldsymbol{\omega} \in S_{h}=[-\pi / h, \pi / h]^{2} .
$$

This is the analogous process to the Fourier decomposition step of von Neumann stability analysis for PDEs.

The continuous Fourier transform (CFT) of the exact RPIE (1) gives the first kind Volterra convolution equation

$$
2 \pi \int_{0}^{t} J_{0}(\omega R) \widehat{u}(\boldsymbol{\omega}, t-R) d R=\widehat{a}(\boldsymbol{\omega}, t) .
$$

$\boldsymbol{\omega}$ is the Fourier frequency, $\boldsymbol{\omega}=|\boldsymbol{\omega}|$, and $J_{0}$ is the first kind Bessel function of order zero. Using a piecewise linear approximation in time for $u$ in (1) corresponds to using the trapezoidal rule to solve (8). This suggests that the coefficients $q_{m}(h \boldsymbol{\omega})$ approximate $2 \pi \Delta t J_{0}\left(\omega t^{m}\right)$, and this is indeed the case [5].

Lemma 1 There exists a constant $C$ independent of $m, h$ and $\boldsymbol{\omega}$ such that

$$
\left|J_{0}\left(\omega t^{m}\right)-\frac{q_{m}(h \boldsymbol{\omega})}{2 \pi \Delta t}\right| \leq C(h \omega)^{2} \quad \text { for all } m \geq 1 \text { and all } \boldsymbol{\omega} \in S_{h} .
$$

Details of the proof are similar to those given in [6] for more standard collocation schemes using exact integration. Our $O\left(h^{2}\right)$ convergence result stems from the second order approximation result given in this Lemma and the stability of the approximate solution. We outline the steps below.

The discrete $\mathrm{L}_{2}$ norm $\|\cdot\|_{h}$ of the error in the approximate solution $U$ satisfies

$$
\left\|U^{n}-u^{n}\right\|_{h} \leq\left\|\tilde{U}^{n}-\widehat{u}^{n}\right\|_{F}+\left\|\widehat{u}^{n}-\tilde{u}^{n}\right\|_{F},
$$

and the discrete Fourier and $\mathrm{L}_{2}$ norms are related through Parseval's identity

$$
\left(h^{2} \sum_{j, k} v_{j, k}^{2}\right)^{1 / 2} \equiv\|\underline{v}\|_{h}=\|\tilde{v}\|_{F} \equiv\left(\frac{1}{4 \pi^{2}} \int_{S_{h}}|\tilde{v}(\boldsymbol{\omega})|^{2} d \boldsymbol{\omega}\right)^{1 / 2} .
$$


It follows from (H1) and [2, Thm. 5] that the difference between the discrete and continuous Fourier transforms of the exact solution $u$ satisfies

$$
\left\|\widehat{u}^{n}-\tilde{u}^{n}\right\|_{F} \leq C h^{s}\|u\|_{H^{s}} \leq C h^{s}\|a\|_{H_{*}^{m+2}\left(0, T ; H^{s+2}\left(\mathbb{R}^{2}\right)\right)},
$$

with $s \geq 4$.

Bounding $\left\|\tilde{U}^{n}-\widehat{u}^{n}\right\|_{F}$ is more complicated. Comparing the CFT (8) of the exact RPIE with the scheme's DFT (7) gives

$$
\sum_{m=1}^{n} q_{n-m}(h \boldsymbol{\omega}) \varepsilon^{m}(\boldsymbol{\omega})=\tilde{a}^{n}(\boldsymbol{\omega})-\widehat{a}^{n}(\boldsymbol{\omega})+e^{n}(\boldsymbol{\omega})
$$

where $\varepsilon^{m}=\tilde{U}^{m}-\widehat{u}^{m}$ is the term we wish to bound. The error term satisfies

$$
\left|e^{n}\right|=\left|\int_{0}^{t^{n}} J_{0}\left(\omega\left(t^{n}-R\right)\right) \widehat{u}(\boldsymbol{\omega}, R) d R-\sum_{m=0}^{n} q_{n-m}(h \boldsymbol{\omega}) \widehat{u}^{m}(\boldsymbol{\omega})\right| \leq F_{1}(\boldsymbol{\omega}) h^{2},
$$

(using Lemma 1 and standard results for the trapezoidal rule: see [6]), where $F_{1} \in L_{2}\left(\mathbb{R}^{2}\right)$, since the exact solution $u \in H_{*}^{2}\left(0, T ; H^{2}\left(\mathbb{R}^{2}\right)\right)$. It is convenient to write $\varepsilon^{n}=\alpha^{n}+\beta^{n}$, and to seek bounds on $\left\|\alpha^{n}\right\|_{F}$ and $\left\|\beta^{n}\right\|_{F}$, where

$$
\sum_{m=1}^{n} q_{n-m}(h \boldsymbol{\omega}) \alpha^{m}(\boldsymbol{\omega})=\tilde{a}^{n}(\boldsymbol{\omega})-\widehat{a}^{n}(\boldsymbol{\omega}), \quad \sum_{m=1}^{n} q_{n-m}(h \boldsymbol{\omega}) \beta^{m}(\boldsymbol{\omega})=e^{n}(\boldsymbol{\omega}) .
$$

It follows from the stability assumption (H2) (see $[5,6])$, that

$$
\left|\alpha^{n}\right| \leq \frac{C}{\Delta t} \sum_{m=1}^{n}\left|\tilde{a}^{m}-\widehat{a}^{m}\right| \quad \text { and } \quad\left|\beta^{n}\right| \leq \frac{C}{\Delta t} \sum_{m=1}^{n}\left|e^{m}\right| \quad \text { for } n=1,2, \ldots
$$

Combining this with (H1) and [2, Thm. 5], we have

$$
\left\|\alpha^{n}\right\|_{F} \leq \frac{C}{\Delta t} \sum_{m=1}^{n}\left\|\tilde{a}^{m}-\widehat{a}^{m}\right\|_{F} \leq C h^{s}\|a\|_{H_{*}^{m+2}\left(0, T ; H^{s+2}\left(\mathbb{R}^{2}\right)\right)}
$$

and combining (14) with (12) gives $\left|\beta^{n}\right| \leq C F_{1}(\boldsymbol{\omega})$.

Unfortunately the bound on $\left|\beta^{n}\right|$ is not precise enough. Returning to (13) when $h \omega$ is "small" $\left(h \omega<C_{0}\right.$ for a sufficiently small constant $\left.C_{0}\right)$, we take the $Z$-transform and use the convolution property to get $Z(\beta)=Z(e) / Z(q)$. It is then possible (but not easy) to obtain an upper bound on $1 /|Z(q)|$ in the $Z$-transform domain, and then invert to get

$$
\left|\beta^{n}\right| \leq F_{2}(\boldsymbol{\omega}) h^{2} \quad \text { for } \omega h \leq C_{0}
$$

in the time domain, where $F_{2} \in L_{2}\left(\mathbb{R}^{2}\right)$ with two more degrees of smoothness in time and one in space on the exact solution $u$ than are already required to get the bound (12). This is guaranteed by (H1). 
Following the arguments used in [12],

$$
\left\|\beta^{n}\right\|_{F}^{2} \leq h^{4} \int_{\text {low }}\left|F_{2}(\boldsymbol{\omega})\right|^{2} d \boldsymbol{\omega}+\int_{\text {high }}\left|\frac{h^{2} \omega^{2}}{C_{0}^{2}} C F_{1}(\boldsymbol{\omega})\right|^{2} d \boldsymbol{\omega} \leq C h^{4}
$$

using bound (15) for "low" frequency $\left(\omega h<C_{0}\right)$ and $\left|\beta^{n}\right| \leq C F_{1}(\boldsymbol{\omega})$ for "high" $\left(\omega h>C_{0} \cap S_{h}\right)$. This requires two extra degrees of smoothness in space on solution $u$ to ensure that $\omega^{2} F_{1}(\boldsymbol{\omega}) \in L^{2}\left(\mathbb{R}^{2}\right)$. Combining the various bounds above yields the result:

Theorem 1. Under hypotheses (H1) and (H2), the global error for the 'polar' approximation scheme satisfies the bound $\left\|\underline{U}^{n}-\underline{u}^{n}\right\|_{h} \leq C h^{2}$ as $h \rightarrow 0$ whenever $t^{n}<T$, where $C$ is a constant.

\section{References}

1. A Bamberger and T Ha-Duong. Formulation variationnelle espace-temps pour le calcul par potentiel retardé de la diffraction d'une onde acoustique (i). Math. Meth. Appl. Sci., 8:405-435, 1986.

2. JH Bramble and SR Hilbert. Estimation of linear functionals on Sobolev spaces with applications to Fourier transforms and spline interpolation. SIAM J. Numer. Anal., 7:112-124, 1970.

3. PJ Davies and DB Duncan. Accuracy and convergence of time marching schemes for RPIEs. (Technical report, University of Dundee), 1995.

4. PJ Davies and DB Duncan. Averaging techniques for time marching schemes for retarded potential integral equations. Appl. Numer. Math., 23:291-310, 1997.

5. PJ Davies and DB Duncan. Numerical stability of collocation schemes for time domain boundary integral equations. Computational Electromagnetics: Proceedings of the GAMM Workshop, Kiel, 2001 eds C Carstensen, S Funken, W Hackbusch, RHW Hoppe and P Monk, Springer, 51-66, 2003.

6. PJ Davies and DB Duncan. Stability and convergence of collocation schemes for retarded potential integral equations. (Under revision.)

7. T Ha-Duong. On Retarded Potential Boundary Integral Equations and their Discretisations. Topics in Computational Wave Propagation eds M Ainsworth, PJ Davies, DB Duncan, PA Martin and BP Rynne, Springer, to appear.

8. Ch Lubich. On the multistep time discretization of linear initial-boundary value problems and their boundary integral equations. Numer. Math., 67:365-389, 1994.

9. A Pujols. Time-dependent integral method for Maxwell equations. In G Cohen, L Halpern and P Joly, editors, Mathematical and Numerical Aspects of Wave Propagation Phenomena, pages 118-126. SIAM, 1991.

10. SM Rao, DR Wilton, and AW Glisson. Electromagnetic scattering by surfaces of arbitrary shape. IEEE Trans. Ant. Prop., 30:409-418, 1982.

11. BP Rynne. Time domain scattering from arbitrary surfaces using the electric field equation. J. Electromag. Waves \& Appl., 5:93-112, 1991.

12. V Thomée. Convergence estimates for semi-discrete Galerkin methods for initialvalue problems. In A Dold and B Eckmann, editors, Numerische, insbesondere approximationstheoretische Behandlung von Funktionalgleichungen (Lecture Notes in Mathematics, 333), pages 243-262. Springer-Verlag, 1973. 